Rev. Mat. Iberoamericana 26 (2010), no. 1, 115-132

\title{
On the Conley decomposition of Mather sets
}

Patrick Bernard

\begin{abstract}
In the context of Mather's theory of Lagrangian systems, we study the decomposition in chain-transitive classes of the Mather invariant sets. As an application, we prove, under appropriate hypotheses, the semi-continuity of the so-called Aubry set as a function of the Lagrangian.
\end{abstract}

In the study of Lagrangian systems, John Mather introduced several invariant sets composed of globally minimizing extremals. He developed methods to construct several orbits undergoing interesting behaviors in phase space under some assumptions on these invariant sets, see [14]. In order to pursue this theory and to apply it on examples, it is necessary to have tools to describe precisely the invariant sets. At least two points of view can be adopted. One can study the invariant set from a purely topological point of view in the style of Conley as compact metric spaces with flows, and study their transitive components. One can also study these set from the point of view of action minimization, and decompose them in invariant subsets that have been called static classes. These points of view are very closely related, but each of them has specific features. For example, understanding the decomposition in static classes is necessary for the variational construction of interesting orbits, while the topological decomposition behaves well under perturbations.

Our goal in the present paper is to explicit the links between these two decompositions. We explain that the topological decomposition is finer than the variational one, and that they coincide for most (but not all) systems. As an application, we prove a result of semi-continuity of the so-called Aubry set as a function of the Lagrangian, under certain non-degeneracy hypotheses.

2000 Mathematics Subject Classification: 37J50, 37B20, 49L25.

Keywords: Semi-continuity of the Aubry set, minimizing measures, chain transitivity. 
The semi-continuity of the Aubry set is a subtle problem, which has remained open for several years, until John Mather gave a counter example, see $\S 18$ in [16]. In the same paper, he also states without proof that semi-continuity holds under appropriate hypotheses. Our result extends the one of Mather. The methods we use are inspired from the recent work of Fathi, Figalli and Rifford, [9].

\section{Introduction}

We have to recall the fundamental constructions of Mather theory before we can state our results. We rapidly expose them without proofs. These proofs are available in $[13,14,10,2,4]$.

We consider a compact manifold without boundary $M$. It is convenient to endow once and for all this manifold with a Riemannian metric. We shall work in the standard framework of Mather theory, and study $C^{2}$ Lagrangians $L(t, q, v): \mathbb{T} \times T M \longrightarrow \mathbb{R}$, where $\mathbb{T}=\mathbb{R} / \mathbb{Z}$. Given $t \in \mathbb{R}$, we will also denote by $t$ the associated element of $\mathbb{T}$. Given $\tau \in \mathbb{T}$, we will also denote by $\tau$ the corresponding element of $[0,1[$. The main object of study is the dynamics of minimizing extremals of $L$. An absolutely continuous curve $q(t): I \longrightarrow M$ is called a minimizing extremal if, for each $t_{0} \leqslant t_{1}$ in $I$ and each absolutely continuous curve $\gamma(t):\left[t_{0}, t_{1}\right] \longrightarrow M$, satisfying $\gamma\left(t_{0}\right)=q\left(t_{0}\right)$ and $\gamma\left(t_{1}\right)=$ $q\left(t_{1}\right)$, we have

$$
\int_{t_{0}}^{t_{1}} L(t, q(t), \dot{q}(t)) d t \leqslant \int_{t_{0}}^{t_{1}} L(t, \gamma(t), \dot{\gamma}(t)) d t .
$$

The curve $q(t)$ is called a locally minimizing extremal if each time $t$ is contained in the interior of an interval $J$ such that $q$ is a minimizing extremal on $J$. We assume

Convexity: For each $(t, x) \in \mathbb{T} \times M$, the function $v \longrightarrow L(t, x, v)$ is convex, has positive definite Hessian at each point, and is superlinear. In short, for each $(t, x) \in \mathbb{T} \times M$, we have

$\partial_{v}^{2} L(t, x, v)>0, \forall v \in T_{x} M$ and $\lim _{\lambda \rightarrow \infty}(L(t, x, \lambda v) / \lambda)=\infty \forall v \in T_{x} M-\{0\}$.

Under the convexity hypothesis, there exists a vector-field $E_{L}$ on $\mathbb{T} \times T M$, the Euler-Lagrange vector-field, such that a curve $q(t)$ is a local minimizing extremal if and only if the associated curve $t \longmapsto(t, q(t), \dot{q}(t))$ is an integral curve of $E_{L}$. We assume

Completeness: The Euler-Lagrange vector-field has a complete flow $\phi^{t}$ on $\mathbb{T} \times T M$. 
A $C^{2}$ Lagrangian satisfying convexity and completeness will be called a Tonelli Lagrangian in the sequel. It is useful to define the function $A_{L}$ : $\mathbb{R} \times M \times \mathbb{R} \times M \longrightarrow \mathbb{R}$ by the expression

$$
A_{L}(S, q ; T, r):=\min _{\gamma(S)=q, \gamma(T)=r} \int_{S}^{T} L(t, \gamma(t), \dot{\gamma}(t)) d t
$$

where the minimum is taken on the set of $C^{1}$ curves $\gamma(t):[S, T] \longrightarrow M$ which satisfy $\gamma(S)=q$ and $\gamma(T)=r$. The existence of the minimum for a Tonelli Lagrangian is a standard result derived from Tonelli's work. It is known that there exists a unique constant $\alpha(L)$ such that the function $\tilde{A}_{L}(S, q, T, r)+(T-S) \alpha(L)$ is bounded on $\{T \geqslant S+1\}$. This constant is sometimes called the Mañé critical value, although it was first introduced by Mather in [13]. Most of the dynamics of locally minimizing orbits is encoded in the function $h_{L}: \mathbb{T} \times M \times \mathbb{T} \times M \longrightarrow \mathbb{R}$ defined, following Mather, by

$$
h_{L}(\tau, q ; \theta, r):=\liminf _{\mathbb{N} \ni T \longrightarrow \infty}\left(A_{L}(\tau, q ; \theta+T, r)+(T+\theta-\tau) \alpha(L)\right) .
$$

The function $h_{L}$ is Lipschitz continuous (and semi-concave) on $\mathbb{T} \times M \times \mathbb{T} \times M$. A function $u: \mathbb{T} \times M \longrightarrow \mathbb{R}$ is said dominated by $L$ if

$$
u(t, q(t))-u(s, q(s)) \leqslant \int_{s}^{t} L(\sigma, q(\sigma), \dot{q}(\sigma))+\alpha(L) d \sigma
$$

for each curve $q(\sigma) \in C^{1}(\mathbb{R}, M)$ and each $s<t$ in $\mathbb{R}$. This implies that

$$
u(y)-u(x) \leqslant h_{L}(x, y) \quad \forall x, y \in \mathbb{T} \times M .
$$

As was noticed by Albert Fathi, the relevance of dominated functions is that there are invariant sets of the Euler-Lagrange flow naturally associated to them. In order to define these sets, it is necessary first to define, following Fathi, the notion of calibrated curve. A curve $q(t): I \longrightarrow M$ is said calibrated by the dominated function $u$ if, for each $s<t$ in $I$, the equality holds in (D). It is clear that calibrated curves are minimizing extremals. For each dominated function $u$, we define the set

$$
\tilde{\mathcal{I}}(L, u) \subset \mathbb{T} \times T M
$$

as follows: $\tilde{\mathcal{I}}(L, u)$ is the set of points $(\tau, q, v)$ such that there exists a calibrated curve $q(s): \mathbb{R} \longrightarrow M$ satisfying $(\tau, q, v)=(\tau, q(\tau), \dot{q}(\tau))$. It is known that $\tilde{\mathcal{I}}(L, u)$ is a compact invariant set of the Euler-Lagrange flow. The projection $\pi:(t, x, v) \longmapsto(t, x)$ induces a bi-Lipschitz homeomorphism between $\tilde{\mathcal{I}}(L, u)$ and its image $\mathcal{I}(L, u) \subset \mathbb{T} \times M$. We shall always endow $\tilde{\mathcal{I}}(L, u)$ with the flow induced from the Euler-Lagrange flow, and $\mathcal{I}(L, u)$ with the conjugated flow. 
The function $u$ is called a Weak KAM solution if it is dominated and if, in addition, for each point $(\tau, q) \in \mathbb{T} \times M$, there exists a calibrated curve $q(s):(-\infty, \tau] \longrightarrow M$ such that $q(\tau)=q$. Given a Weak KAM solution $u$, we define the set

$$
\overline{\mathcal{G}}(L, u) \subset \mathbb{T} \times T M
$$

as the set of points $(\tau, q, v)$ such that there exists a calibrated curve $q(s)$ : $(-\infty, \tau] \longrightarrow M$ satisfying $q(\tau)=q$ and $\dot{q}(\tau)=v$. The set $\overline{\mathcal{G}}(L, u)$ is compact and negatively invariant, so that the Euler-Lagrange flow defines on it a semi-flow (with negative times). Note also that $\pi(\overline{\mathcal{G}}(L, u))=\mathbb{T} \times M$ and that

$$
\tilde{\mathcal{I}}(L, u)=\bigcap_{t \leqslant 0} \phi^{t}(\overline{\mathcal{G}}(L, u)) .
$$

It is known that, for each $x_{0} \in M$, the function $x \longmapsto h_{L}\left(x_{0}, x\right)$ is a Weak KAM solution.

The set

$$
\tilde{\mathcal{A}}(L):=\bigcap \tilde{\mathcal{I}}(L, u)
$$

is not empty, where the intersection is taken on the set of all dominated functions, or equivalently on the set of all Weak KAM solutions. This is the definition of Fathi of a set previously introduced by John Mather in [15], and called the Aubry set. It is clearly compact and invariant, we shall always endow it with the Euler-Lagrange flow. The projection $\pi$ restricted to $\tilde{\mathcal{A}}(L)$ is a bi-Lipschitz homeomorphism into its image $\mathcal{A}(L)$. We endow this image with the conjugated flow. Let us recall from now on that Fathi proved the existence of a Weak KAM solution $u$ such that $\mathcal{I}(L, u)=\mathcal{A}(L)$. John Mather noticed that the function

$$
d_{L}(x, y):=h_{L}(x, y)+h_{L}(y, x)
$$

is a pseudo-metric on $\mathcal{A}(L)$. Indeed, it is known that $d_{L}(x, y) \geqslant 0$ for each $x$ and $y$ in $\mathbb{T} \times M$, and that $d_{L}(x, x)=0$ if and only if $x$ belongs to $\mathcal{A}(L)$. The function $d_{L}$ is symmetric and satisfies the triangle inequality, but there may exist points $x \neq y$ such that $d_{L}(x, y)=0$. The relation $\mathfrak{R}$ on $\mathcal{A}$ defined by

$$
x \Re y \Longleftrightarrow d_{L}(x, y)=0
$$

is then an equivalence relation on $\mathcal{A}(L)$. The equivalence classes are called the static classes. They are compact invariant subsets of $\mathcal{A}(L)$. Note that the pseudo-metric $d_{L}$ descends to a metric, that we still denote by $d_{L}$, on the set $\dot{\mathcal{A}}(L)$ of static classes. The set $\dot{\mathcal{A}}(L)$, endowed with the metric $d_{L}$ has been called by Mather the quotient Aubry set. It is a compact metric space. 
The set

$$
\tilde{\mathcal{N}}(L):=\bigcup \tilde{\mathcal{I}}(L, u)
$$

is compact and invariant, where the union is taken on the set of all dominated functions, or equivalently on the set of all Weak KAM solutions. This is the definition of Fathi of a set previously introduced by John Mather [15], and called the Mañé set. The $\alpha$ and $\omega$-limits of orbits of the Mañé set are contained in the Aubry set, see for example [2] or [15]. More is true: given an orbit of the Mañé set, there exists a static class which contain all its $\alpha$-limit points and a static class which contain all its $\omega$-limit points. These static classes are equal if and only if the orbit is contained in the Aubry set.

For each Weak KAM solution $u$, we define the relation $x \Re_{u} y$ on $\mathbb{T} \times M$ by

$$
x \Re_{u} y \Longleftrightarrow u(y)-u(x)=h_{L}(x, y) .
$$

This relation is transitive. Indeed, we have $x \Re_{u} y \Longleftrightarrow u(y)-u(x) \geqslant h_{L}(x, y)$ (the converse inequality always holds). If $x_{0} \mathfrak{R}_{u} x_{1}$ and $x_{1} \mathfrak{R}_{u} x_{2}$, then we have

$$
u\left(x_{2}\right)-u\left(x_{1}\right) \geqslant h_{L}\left(x_{0}, x_{1}\right)+h_{L}\left(x_{1}, x_{2}\right) \geqslant h_{L}\left(x_{0}, x_{2}\right)
$$

so that $x_{0} \Re_{u} x_{2}$. The Aubry set $\mathcal{A}(L)$ is the set of points $x \in \mathbb{T} \times M$ such that $x \mathfrak{R}_{u} x$. The symmetrized relation is nothing but $\mathfrak{R}$ :

$\left(x \Re_{u} y\right.$ and $\left.y \Re_{u} x\right) \Longleftrightarrow\left(x \in \mathcal{A}(L)\right.$ and $y \in \mathcal{A}(L)$ and $\left.d_{L}(x, y)=0\right)$.

We denote by $\mathfrak{C}_{u}$ the relation of chain-connection on $\mathcal{I}(L, u)$, see Appendix. We use the same symbol for the relation of chain connection in $\tilde{\mathcal{I}}(L, u)$ and in $\overline{\mathcal{G}}(L, u)$ (these relations coincide on $\tilde{\mathcal{I}}(L, u)$ by Lemma 18 of the Appendix).

Proposition 1. For each Weak KAM solution $u$, and for $x$ and $y$ in $\mathcal{I}(L, u)$, we have

$$
x \Re_{u} y \Longrightarrow x \mathfrak{C}_{u} y .
$$

More precisely, given two points $x$ and $y$ in $\mathbb{T} \times M$, the relation $x \mathfrak{R}_{u} y$ implies that $x \in \mathcal{I}(L, u)$ and that there exists points $\tilde{x}$ and $\tilde{y}$ above $x$ and $y$ in $\overline{\mathcal{G}}(L, u)$ such that $\tilde{x} \mathfrak{C}_{u} \tilde{y}$.

The proof will be given in Section 2. The following statement is due tu Mañé, see [12], and a proof can be found in [10].

Corollary 2. The Mañé set $\tilde{\mathcal{N}}(L)$ is chain transitive. The Aubry set $\mathcal{A}(L)$ is chain recurrent. Each static class is chain transitive in $\mathcal{A}(L)$.

We give a proof as an application of Proposition 1. 
Proof. In order to prove the chain recurrence of the Aubry set, let us first recall that there exists a weak KAM solution $u$ such that $\mathcal{I}(L, u)=\mathcal{A}(L)$. For this function $u$, we have $x \mathfrak{R}_{u} x \Rightarrow x \mathfrak{C}_{u} x$, which precisely says that the point $x$ is chain-recurrent in $\mathcal{A}(L)$. If $x$ and $y$ belong to the same static class, then we have, still with the same function $u$, that $x \mathfrak{R}_{u} y$, and therefore $x \mathfrak{C}_{u} y$. Finally, the chain transitivity of the Mañé set can be proved in several steps. First, let $x$ and $y$ be points of $\mathcal{A}(L)$. Then, taking $u=h(x,$.$) , we see$ that $x \Re_{u} y$, and therefore $x \mathfrak{C}_{u} y$. As a consequence, $x$ and $y$ can be connected by chains in $\mathcal{I}(L, u)$, and therefore the points $\tilde{x}$ and $\tilde{y}$ which are the points of $\tilde{\mathcal{I}}(L, u)$ above $x$ and $y$ can be connected by chains in $\tilde{\mathcal{N}}(L)$. Now since every point $\tilde{x} \in \tilde{\mathcal{N}}(L)$ has its $\omega$-limit contained in $\tilde{\mathcal{A}}(L)$, there exists a point $\tilde{\omega} \in \tilde{\mathcal{A}}(L)$ such that $\tilde{x} \mathfrak{C}_{\tilde{\mathcal{N}}} \tilde{\omega}$. In the same way, for each $\tilde{y} \in \tilde{\mathcal{N}}(L)$, there exists a point $\tilde{\alpha} \in \tilde{\mathcal{A}}$ such that $\tilde{\alpha} \mathfrak{C}_{\tilde{\mathcal{N}}} y$. By transitivity of the relation $\mathfrak{C}_{\tilde{\mathcal{N}}}$, we conclude that $\tilde{x} \mathfrak{C}_{\tilde{\mathcal{N}}} \tilde{y}$.

It is known that the converse to Proposition 1 does not hold in general. However, it holds in many examples, and has interesting consequences, that we now describe.

Definition 3. We say that the Lagrangian L satisfies the coincidence hypothesis if, for each Weak KAM solution $u$, the relations $\mathfrak{C}_{u}$ and $\mathfrak{R}_{u}$ coincide on $\mathcal{I}(L, u)$.

By well-known properties of the Conley decomposition of flows recalled in the Appendix, we obtain:

Proposition 4. If $L$ satisfies the coincidence hypothesis, then, for each Weak KAM solution u, the Aubry set is precisely the chain-recurrent set of $\overline{\mathcal{G}}(L, u)$. The static classes are the chain transitive components of $\mathcal{A}(L)$, they are also the connected components of $\mathcal{A}(L)$. The quotient Aubry set is totally disconnected.

The coincidence hypothesis also has as a consequence the semi-continuity of the Aubry set. In order to be more precise, we now introduce a notion of convergence for Tonelli Lagrangians. The sequence $L_{k}$ of Tonelli Lagrangians is said to converge to $L$ if $\left\{L_{k}\right\}$ is a uniform family of Tonelli Lagrangians (see definition in Section 2), and if $L_{k}$ converge to $L$ uniformly on compact sets as $k \longrightarrow \infty$. We shall study this convergence in Section 2 .

Theorem 1. Let $L$ be a Tonelli Lagrangian satisfying the coincidence hypothesis. Let $L_{k}$ be a sequence of Tonelli Lagrangians converging to $L$. Let $U$ be a neighborhood of $\tilde{\mathcal{A}}(L)$ in $\mathbb{T} \times T M$. Then, there exists $k_{0}$ such that $\tilde{\mathcal{A}}\left(L_{k}\right) \subset U$ for each $k \geqslant k_{0}$.

In general (without the coincidence hypothesis), the set $\lim \sup \tilde{\mathcal{A}}\left(L_{k}\right)$ is contained in the chain recurrent set of $\tilde{\mathcal{N}}(L)$ (and more precisely in the union of the chain recurrent sets of $\tilde{\mathcal{I}}(L, u))$. 
John Mather has stated this result without proof in [16] under the hypothesis that the quotient Aubry set $\dot{\mathcal{A}}(L)$ has vanishing 1-dimensional Hausdorff measure. We shall see that this hypothesis of Mather implies the coincidence hypothesis hence the statement above is stronger than Mather's one. A very partial version of Theorem 1 was proved earlier in [1]. The examples described in [16] show that the semi-continuity of the Aubry set with respect to the Lagrangian is not always true.

In order to extract more information from the constructions presented above, Mather noticed that, when $\omega$ is a closed one-form on $M$, the Lagrangian

$$
(L+\omega)(t, x, v):=L(t, x, v)+\omega_{x}(v)
$$

has the same Euler-Lagrange extremals than $L$, but not the same Aubry set. The Aubry set of $L+\omega$ depends only on the De Rham cohomology class of $\omega$ in $H^{1}(M, \mathbb{R})$. Given $c \in H^{1}(M, \mathbb{R})$, we denote by $\mathcal{A}(L+c)$ the Aubry set of $L+\omega$, where $\omega$ is any closed form of cohomology $c$. The semi-continuity of the Aubry set with respect to the cohomology $c$ is an open question, but we have:

Corollary 5. Given a Tonelli Lagrangian L, the set-valued map

$$
H^{1}(M, \mathbb{R}) \ni c \longmapsto \tilde{\mathcal{A}}(L+c)
$$

is upper-semicontinuous at every point c where $L+c$ satisfies the coincidence hypothesis. If $L$ is generic in the sense of Mañé, then this hypothesis holds for each $c$.

In the sequel, we detail and prove what has been stated above. All is based on basic properties of the Conley decomposition of flows which are recalled in the Appendix. In Section 2, we study the main features of the convergence of Tonelli Lagrangians. We prove Proposition 1 and Theorem 1. Finally, in Section 3, we discuss the hypothesis of coincidence.

\section{Convergence of Tonelli Lagrangians}

We define the notion of convergence that is used throughout the paper, and collect its relevant properties. It is first useful to recall from [4] the notion of uniform family of Tonelli Lagrangians. A Family $\mathbb{L} \subset C^{2}(\mathbb{T} \times T M, \mathbb{R})$ of Tonelli Lagrangians is called uniform if:

(i) There exist two superlinear functions $l_{0}$ and $l_{1}: \mathbb{R}^{+} \longrightarrow \mathbb{R}$ such that each Lagrangian $L$ of the family satisfies $l_{0}\left(|v|_{q}\right) \leqslant L(t, q, v) \leqslant l_{1}\left(|v|_{q}\right)$. 
(ii) There exists an increasing function $K(k): \mathbb{R}^{+} \longrightarrow \mathbb{R}^{+}$such that, if $\phi$ is the Euler-Lagrange flow of a Lagrangian of the Family, then, for each $t \in[-1,1]$, we have

$$
\phi^{t}\left(\left\{|v|_{q} \leqslant k\right\}\right) \subset\left\{|v|_{q} \leqslant K(k)\right\} \subset \mathbb{T} \times T M .
$$

(iii) There exists a finite atlas $\Psi$ of $M$ such that, for each chart $\psi \in \Psi$ and each Lagrangian $L$ of the family, we have $\left\|d^{2}(L \circ T \psi)_{(t, q, v)}\right\| \leqslant K(k)$ for $|v|_{q} \leqslant k$.

The following result is proved in the Appendix of [4]:

Proposition 6. The set of functions $u$ which are Weak KAM solutions of some $L \in \mathbb{L}$ is equi-Lipschitz. For each compact interval $[a, b]$, the set of curves $q(s):[a, b] \longrightarrow M$ which are minimizing extremals for some $L \in \mathbb{L}$ is relatively compact in $C^{1}([a, b], M)$.

The sequence $L_{k}$ of Tonelli Lagrangians is said to converge to $L$ if it forms a uniform family and if $L_{k}$ converges to $L$ uniformly on compact sets.

Lemma 7. If $L_{k} \longrightarrow L$, then $\alpha\left(L_{k}\right) \longrightarrow \alpha(L)$. If $u_{k}$ is a sequence of Weak $K A M$ solutions of $L_{k}$ which converge uniformly to $u$, then $u$ is a Weak KAM solution of $L$.

Proof. Let $u_{k}$ be a sequence of Weak KAM solution of $L_{k}$. Let us take a subsequence such that $\alpha\left(L_{k}\right)$ has a limit $\alpha$ and such that $u_{k}$ has a uniform limit $u$. Taking the limit in the inequality

$$
u_{k}(t, q(t))-u_{k}(s, q(s)) \leqslant \int_{s}^{t} L_{k}(\sigma, q(\sigma), \dot{q}(\sigma))+\alpha_{k}(L) d \sigma
$$

we get

$$
u(t, q(t))-u(s, q(s)) \leqslant \int_{s}^{t} L(\sigma, q(\sigma), \dot{q}(\sigma))+\alpha d \sigma
$$

for each curve $q(\sigma) \in C^{1}([s, t], M)$. This implies that $\alpha \geqslant \alpha(L)$. For each $x=(\tau, q) \in \mathbb{T} \times M$, let us now consider a sequence $q_{k} \in C^{1}((-\infty, \tau], M)$ such that $q_{k}(\tau)=q$ and such that

$$
u_{k}\left(\tau, q_{k}(\tau)\right)-u_{k}\left(s, q_{k}(s)\right)=\int_{s}^{\tau} L_{k}\left(\sigma, q_{k}(\sigma), \dot{q}_{k}(\sigma)\right)+\alpha_{k}(L) d \sigma
$$

for each $s<\tau$. This sequence has a subsequence which converges in $C_{l o c}^{1}((-\infty, \tau], M)$ to a limit $q(t)$, which satisfies

$$
u(\tau, q(\tau))-u(s, q(s))=\int_{s}^{\tau} L(\sigma, q(\sigma), \dot{q}(\sigma))+\alpha d \sigma
$$

for each $s<\tau$. The existence of such a curve implies that $\alpha(L)=\alpha$ and that $u$ is a Weak KAM solution of $L$. 
Proof of Proposition 1. Let us fix two points $x=(\tau, q)$ and $y=(\theta, r)$ in $\mathbb{T} \times M$ such that $h_{L}(x, y)=u(y)-u(x)$. Let $q_{k}(t):\left[\tau, \theta+T_{k}\right] \longrightarrow M$ be a sequence of minimizing trajectories of $L$ such that $\mathbb{N} \ni T_{k} \longrightarrow \infty, q_{k}(\tau)=q$, $q_{k}\left(T_{k}+\theta\right)=r$ and

$$
\int_{\tau}^{\theta+T_{k}} L\left(t, q_{k}(t), \dot{q}_{k}(t)\right)+\alpha(L) d t \longrightarrow h_{L}(x, y)=u(y)-u(x) .
$$

Let us consider a sequence $S_{k} \in\left[\tau, \theta+T_{k}\right]$, and assume that either $S_{k}=\tau$ (case a) or $S_{k}=\theta+T_{k}$ (case b), or both $S_{k}$ and $T_{k}-S_{k}$ converge to $\infty$ (case c). By taking a subsequence, we can assume that $S_{k} \bmod 1$ has a limit $S$ in $\mathbb{T}$, and that the sequence $q_{k}\left(t+S_{k}\right)$ converge in $C_{l o c}^{1}(I, M)$ to a limit $q(t)$ where $I=[0, \infty)$ in case a, $I=(-\infty, 0]$ in case $\mathrm{b}$, and $I=\mathbb{R}$ in case c.

We have, for all $s<t$ in the interior of $I$, the equality

$\left.u\left(t+S_{k}, q_{k}\left(t+S_{k}\right)\right)\right)-u\left(s+S_{k}, q_{k}\left(s+S_{k}\right)\right)=\int_{s+S_{k}}^{t+S_{k}} L\left(\sigma, q_{k}(\sigma), \dot{q}_{k}(\sigma)\right)+\alpha(L) d \sigma$

when $k$ is large enough. At the limit, we get

$$
u(t+S, q(t))-u(s+S, q(s))=\int_{s+S}^{t+S} L(\sigma, q(\sigma-S), \dot{q}(\sigma-S))+\alpha(L) d \sigma .
$$

In other words, the limit curve $t \longmapsto q(t-S)$ is calibrated by $u$ on its interval of definition. In case a, the limit curve $q(t-\tau):[\tau, \infty) \longrightarrow M$ satisfies $q(\tau-\tau)=q$. This implies that the point $x$ belongs to $\mathcal{I}(L, u)$.

Moreover, what we have proved implies, in the terminology of the Appendix, that the sequence of curves

$$
\tilde{x}_{k}(t)=\left(t, q_{k}(t), \dot{q}_{k}(t)\right):\left[\tau, \theta+T_{k}\right] \longrightarrow \mathbb{T} \times T M
$$

accumulate on $\overline{\mathcal{G}}(L, u)$. We conclude that $\tilde{x} \mathfrak{C}_{u} \tilde{y}$, where $\tilde{x}=\lim \tilde{x}(0)$, and $\tilde{y}=\lim \tilde{y}\left(\theta+T_{k}\right)$. Note that $\tilde{x}=(\tau, q, \dot{q}(0))$, where $q(t)$ is the limit curve obtained in case a, and $\tilde{y}=(\theta, r, \dot{q}(0))$, where $q(t)$ is the limit curve obtained in case b. We have $\tilde{x} \in \tilde{\mathcal{I}}(L, u)$ and $\tilde{y} \in \overline{\mathcal{G}}(L, u)$.

Lemma 8. Assume that $L_{k} \longrightarrow L$ and that $u_{k}$ is a sequence of Weak KAM solutions of $L_{k}$ such that $u_{k} \longrightarrow u$ in $C^{0}$. Then the semi-flows $\overline{\mathcal{G}}\left(L_{k}, u_{k}\right)$ accumulate on $\overline{\mathcal{G}}(L, u)$, see definition in the Appendix. If the compact sets $\overline{\mathcal{G}}\left(L_{k}, u_{k}\right)$ converge, for the Hausdorff metric, to a limit $\tilde{\mathcal{K}}$, then $\tilde{\mathcal{K}}$ is a negatively invariant subset of $\overline{\mathcal{G}}(L, u)$ such that $\pi(\tilde{\mathcal{K}})=\mathbb{T} \times M$. 
Proof. It is enough to prove that, if $\tilde{x}_{k}(t):(-\infty, 0] \longrightarrow \mathbb{T} \times T M$ is a sequence of orbits of $\overline{\mathcal{G}}\left(L_{k}, u_{k}\right)$, then it has a subsequence which converges uniformly to an orbit of $\overline{\mathcal{G}}(L, u)$. By definition of the semi-flow of $\overline{\mathcal{G}}\left(L_{k}, u_{k}\right)$, we have $\tilde{x}_{k}(t)=\left(t, q_{k}\left(t+\tau_{k}\right), \dot{q}_{k}\left(t+\tau_{k}\right)\right)$, where $\tau_{k} \in \mathbb{T}$ and $q_{k} \in C^{2}\left(\left(-\infty, \tau_{k}\right], M\right)$ satisfy

$$
u_{k}\left(\tau_{k}, q_{k}\left(\tau_{k}\right)\right)-u_{k}\left(\tau_{k}-T, q_{k}\left(\tau_{k}-T\right)\right)=\int_{\tau_{k}-T}^{\tau_{k}} L_{k}\left(t, q_{k}(t), \dot{q}_{k}(t)\right)+\alpha\left(L_{k}\right) d t
$$

for each $T \geqslant 0$. We can assume that $\tau_{k}$ has a limit $\tau$ and that $q_{k}$ converges in $C_{l o c}^{1}((-\infty, \tau[, M)$ to a curve $q(t)$. For each $T \geqslant 0$ and $\epsilon>0$, we have, for $k$ large enough,

$u\left(\tau-\epsilon, q_{k}(\tau-\epsilon)\right)-u\left(\tau-T, q_{k}(\tau-T)\right)=\int_{\tau-T}^{\tau-\epsilon} L_{k}\left(t, q_{k}(t), \dot{q}_{k}(t)\right)+\alpha\left(L_{k}\right) d t$.

By taking the limits $k \longrightarrow \infty$ and then $\epsilon \longrightarrow 0$, we get

$$
u(\tau, q(\tau))-u(\tau-T, q(\tau-T))=\int_{\tau-T}^{\tau} L(t, q(t), \dot{q}(t))+\alpha(L) d t .
$$

This equality means that $t \longmapsto(t, q(t+\tau), \dot{q}(t+\tau))$ is an orbit of $\overline{\mathcal{G}}(L, u)$. If the compact sets $\overline{\mathcal{G}}\left(L_{k}, u_{k}\right)$ converge, for the Hausdorff metric, to a limit $\tilde{\mathcal{K}}$, then, what we have just done shows that $\tilde{\mathcal{K}} \subset \overline{\mathcal{G}}(L, u)$. We have $\pi(\tilde{\mathcal{K}})=$ $\mathbb{T} \times M$ because $\pi\left(\overline{\mathcal{G}}\left(L_{k}, u_{k}\right)\right)=\mathbb{T} \times M$.

An interesting consequence is that, in the context above, if $\tilde{x}_{k} \in \overline{\mathcal{G}}\left(L_{k}, u_{k}\right)$ and $\tilde{y}_{k} \in \overline{\mathcal{G}}\left(L_{k}, u_{k}\right)$ satisfy $\tilde{x}_{k} \longrightarrow \tilde{x}, \tilde{y}_{k} \longrightarrow \tilde{y}$ and $\tilde{x}_{k} \mathfrak{C}_{u_{k}} \tilde{y}_{k}$, then $\tilde{x} \mathfrak{C}_{u} \tilde{y}$.

Lemma 9. Assume that $L_{k} \longrightarrow L$ and that $u_{k}$ is a sequence of Weak $K A M$ solutions of $L_{k}$, such that $u_{k} \longrightarrow u$ in $C^{0}$. Assume furthermore that $\tilde{\mathcal{I}}\left(L_{k}, u_{k}\right)$ has a limit $\tilde{\mathcal{K}}$ for the Hausdorff distance of compact sets. Then, $\tilde{\mathcal{K}}$ is a compact invariant subset of $\tilde{\mathcal{I}}(L, u)$. If $x_{k} \in \mathcal{I}\left(L_{k}, u_{k}\right)$ and $y_{k} \in \mathcal{I}\left(L_{k}, u_{k}\right)$ are sequences such that $x_{k} \longrightarrow x, y_{k} \longrightarrow y$ and $x_{k} \mathfrak{C}_{u_{k}} y_{k}$, then $x \mathfrak{C}_{u} y$. In addition, for each $y \in \mathbb{T} \times M$, there exists a point $\tilde{y}$ above $y$ in $\overline{\mathcal{G}}(L, u)$ and a point $\tilde{x} \in \tilde{\mathcal{I}}(L, u)$ such that $\tilde{x} \mathfrak{C}_{u} \tilde{y}$.

Proof. We have seen that the semi-flow $\overline{\mathcal{G}}\left(L_{k}, u_{k}\right)$ accumulates on $\overline{\mathcal{G}}(L, u)$. Since $\tilde{\mathcal{I}}\left(L_{k}, u_{k}\right)$ is invariant for this flow, the limit $\tilde{\mathcal{K}}$ is an invariant set of $\overline{\mathcal{G}}(L, u)$, so it is contained in $\tilde{\mathcal{I}}(L, u)$. In order to prove the last statement, let us consider $y \in \mathbb{T} \times M$. There exists a point $\tilde{y}_{k} \in \overline{\mathcal{G}}\left(L_{k}, u_{k}\right)$ above $y$. There exists a sequence $\tilde{x}_{k} \in \tilde{\mathcal{I}}\left(L_{k}, u_{k}\right)$ such that $\tilde{x}_{k} \mathfrak{C}_{u_{k}} \tilde{y}_{k}$. Taking a subsequence, we can assume that the sequences $\tilde{y}_{k}$ and $\tilde{x}_{k}$ have a limits $\tilde{y} \in \overline{\mathcal{G}}(L, u)$ and $\tilde{x} \in \tilde{\mathcal{K}}$. We have $\tilde{x} \mathfrak{C}_{u} \tilde{y}$.

Proof of Theorem 1. It follows from: 
Corollary 10. Assume that $L_{k} \longrightarrow L$ and that $\tilde{\mathcal{A}}\left(L_{k}\right)$ has a limit $\tilde{\mathcal{K}}$ for the Hausdorff metric. Then there exists a Weak KAM solution $u$ of $L$ such that $\tilde{\mathcal{K}}$ is contained in the set of chain recurrent points of $\tilde{\mathcal{I}}(L, u)$. If $L$ satisfies the coincidence hypothesis, we conclude that $\tilde{\mathcal{K}} \subset \tilde{\mathcal{A}}(L, u)$.

Proof. Let $u_{k}$ be a Weak KAM solution of $L_{k}$. The sequence $u_{k}$ is equiLipschitz. By adding appropriate constants, we can suppose that it is also equi-bounded, and that it converges to a limit $u$, which is a Weak KAM solution of $L$. Let us consider a point $\tilde{x} \in \tilde{\mathcal{K}}$. This point is the limit of a sequence $\tilde{x}_{k} \in \mathcal{A}\left(L_{k}\right) \subset \tilde{\mathcal{I}}\left(L_{k}, u_{k}\right)$. We have $\tilde{x}_{k} \mathfrak{C}_{u_{k}} \tilde{x}_{k}$, hence $\tilde{x} \mathfrak{C}_{u} \tilde{x}$.

\section{The coincidence hypothesis}

Let us mention several hypotheses of non-degeneracy that appear in the literature. The discussions in the present section are elaborations on the recent work of Fathi, Figalli and Rifford [9].

1. The quotient Aubry set is finite

2. The quotient Aubry set has Hausdorff dimension zero.

3. The quotient Aubry set has zero 1-Hausdorff measure.

4. For each pair $u, v$ of Weak KAM solutions, the image $(u-v)(\mathcal{A}(L))$ has Lebesgue measure zero in $\mathbb{R}$.

5. For each pair $u, v$ of Weak KAM solutions, the image $(u-v)(\mathcal{A}(L))$ is totally disconnected in $\mathbb{R}$.

6. The coincidence hypothesis holds

7. The quotient Aubry set is totally disconnected (which is equivalent to the statement that the static classes are the connected components of the Aubry set).

The hypotheses 4 . and 5. come from [9].

\section{Theorem 2.}

$$
1 \Rightarrow 2 \Rightarrow 3 \Rightarrow 4 \Leftrightarrow 5 \Rightarrow 6 \Rightarrow 7 \text {. }
$$

The implication $5 \Rightarrow 4$ was pointed out to the author by Albert Fathi.

Proof. It is obvious that $1 \Rightarrow 2 \Rightarrow 3$. and that $4 \Rightarrow 5$. In order to prove that $3 \Rightarrow 4$, it is sufficient to notice that the difference $(u-v)$ is Lipschitz with respect to the pseudo-metric $d_{L}$ on $\mathcal{A}(L)$. Assuming 3 , this implies that the image $(u-v)(\mathcal{A}(L))$ has zero 1-Hausdorff measure in $\mathbb{R}$ and therefore zero Lebesgue measure, see [9] for more details. We have already seen that $6 \Rightarrow 7$. 
Let us prove that $5 \Rightarrow 6$. The method is inspired from [9]. We assume 5 , consider a Weak KAM solution $u$ of $L$ and two points $x$ and $y$ in $\mathcal{I}(L, u)$ such that $x \mathfrak{C}_{u} y$. We denote by $\varphi^{t}$ the natural flow on $\mathcal{I}(L, u)$. We want to prove that $u(y)-u(x)=h(x, y)$, or equivalently, setting $w(z):=h(x, z)-u(z)$, that $w(y)=w(x)$. Contradicting this conclusion, we assume that $w(y)>w(x)$ (note that we always have $w(y) \geqslant w(x)$ ). Since we assumed 5 , and since $w$ is a difference of Weak KAM solutions, there exist real numbers $a$ and $b$ such that $w(x)<a<b<w(y)$ and such that $w(\mathcal{A}(L)) \cap[a, b]=\emptyset$. The function $w$ is non-increasing on the orbits of $\varphi^{t}$. This can be seen as follows: If $x(t)$ is an orbit of this flow, and if $s<t$, then

$$
u(x(t))-u(x(s)))=\int_{s}^{t} L(\sigma, x(\sigma), \dot{x}(\sigma))+\alpha(L) d \sigma
$$

while

$$
\left.h_{L}(x, x(t))-h_{L}(x, x(s))\right) \leqslant \int_{s}^{t} L(\sigma, x(\sigma), \dot{x}(\sigma))+\alpha(L) d \sigma .
$$

We conclude that the compact sets $\mathcal{I}(L, u) \cap\{w \leqslant b\}$ and $\mathcal{I}(L, u) \cap\{w \leqslant a\}$ are positively invariant by the flow. It is known that each orbits of $\mathcal{I}(L, u)$ is $\omega$-asymptotic to $\mathcal{A}(L)$. All the orbits starting in $\mathcal{I}(L, u) \cap\{w \leqslant b\}$ are thus $\omega$-asymptotic to $\mathcal{A}(L) \cap\{w \leqslant b\}=\mathcal{A}(L) \cap\{w<a\}$. As a consequence, there exists $T>0$ such that

$$
\varphi^{T}(\mathcal{I}(L, u) \cap\{w \leqslant b\}) \subset \mathcal{I}(L, u) \cap\{w \leqslant a\} .
$$

Let us pick $\epsilon>0$ such that, for each points $z$ and $z^{\prime}$ in $\mathcal{I}(L, u)$ satisfying $w(z) \leqslant a$ and $d\left(z, z^{\prime}\right) \leqslant \epsilon$, we have $w\left(z^{\prime}\right)<b$. We claim that no $(\epsilon, T)$-chain can connect $x$ and $y$. Indeed, let $x(t):[0, S] \longrightarrow M$ be an $(\epsilon, T)$-chain such that $x(0)=x$. We claim that $w(x(t))<b$ for each $t$. Therefore, it is not possible to have $x(S)=y$. In order to prove the claim let us denote by $\tau_{i}$ the jump times. We have $w(x(t)) \leqslant w\left(x\left(\tau_{i}^{+}\right)\right)$for all $t \in\left[\tau_{i}, \tau_{i+1}\right.$. So it is enough to prove that $w\left(x\left(\left(\tau_{i}^{+}\right)\right)<b\right.$ for each $i$. This can be proved by recurrence. If $w\left(x\left(\left(\tau_{i}^{+}\right)\right)<b\right.$, then, since $\tau_{i+1} \geqslant \tau_{i}+T$, we have

$$
x\left(\tau_{i+1}^{-}\right) \in \varphi^{T}(\mathcal{I}(L, u) \cap\{w \leqslant b\}) \subset \mathcal{I}(L, u) \cap\{w \leqslant a\} .
$$

But then, since $\left.d\left(x\left(\tau_{i+1}^{-}\right), \tau_{i+1}^{+}\right)\right) \leqslant \epsilon$, the way we have chosen $\epsilon$ guarantees that $w\left(x\left(\tau_{i+1}^{+}\right)\right)<b$. This ends the proof of $5 \Rightarrow 6$.

Finally, the implication $5 \Rightarrow 4$. follows from the next Lemma (a courtesy of Albert Fathi).

Lemma 11. If there exist two weak KAM solutions $u$ and $v$ such that $(v-u)(\mathcal{A}(L))$ has positive Lebesgue measure, then there exists a third solution $w$ such that $(w-u)(\mathcal{A}(L))$ is a non-trivial interval. 
Proof. It is useful to recall that a function $w: \mathcal{A}(L) \longrightarrow \mathbb{R}$ is the restriction to $\mathcal{A}(L)$ of a weak KAM solution if and only if it satisfies

$$
w(y)-w(x) \leqslant h_{L}(x, y)
$$

for each $x$ and $y$ in $\mathcal{A}(L)$, see [8] and [5]. Indeed, such a function can be extended to a weak KAM solution on $\mathbb{T} \times M$ by the formula

$$
w(x)=\min _{a \in \mathcal{A}(L)}\left(w(a)+h_{L}(a, x)\right) .
$$

Let us denote by $A \subset \mathbb{R}$ the set $A:=(v-u)(\mathcal{A}(L))$, by $1_{A}(t)$ the characteristic function of $A$, and by $\theta_{A}(t)$ a primitive of $1_{A}(t)$. Then, we define the function $w$ on $\mathcal{A}(L)$ by

$$
w:=u+\theta_{A} \circ(v-u) .
$$

It is not hard to see that $(w-u)(\mathcal{A}(L))=\theta_{A}(A)$ is a non-trivial interval (assuming that $A$ has positive Lebesgue measure). So we have to prove that $w$ can be extended to a weak KAM solution, or equivalently, that (3.1) holds on $\mathcal{A}(L)$. Assume first that $(v-u)(y) \leqslant(v-u)(x)$. Then, $\theta_{A}((v-u)(y)) \leqslant$ $\theta_{A}((v-u)(x))$ so that

$$
w(y)-w(x) \leqslant u(y)-u(x) \leqslant h_{L}(x, y) .
$$

In the other case, when $(v-u)(y) \geqslant(v-u)(x)$, we have, using that $\theta_{A}$ is 1-Lipschitz,

$w(y)-w(x) \leqslant u(y)-u(x)+(v-u)(y)-(v-u)(x)=v(y)-v(x) \leqslant h_{L}(x, y)$.

This ends the proof of Lemma 11.

John Mather produced in [16] the example of a Lagrangian violating 6. However, such examples are rather exceptional. Indeed, Ricardo Mañé proved in [11] that the property of having only one static class is generic in the following sense (generic in the sense of Mañé):

For each Tonelli Lagrangian $L$, there exists a dense $G_{\delta}$ set $\mathcal{O} \subset C^{\infty}(\mathbb{T} \times M)$ of potentials such that the property is satisfied by the Lagrangian $L(t, x, v)+$ $g(t, x)$ for each $g \in \mathcal{O}$.

Moreover, we proved in [6] that the following property is generic in the sense of Mañé (and, in a certain sense, satisfied outside of a singular set of infinite codimension, see [6]):

For each $c \in H^{1}(M, \mathbb{R})$, the Lagrangian $L+c$ satisfies 1 .

It is even believed that the property 3 may hold for all smooth Lagrangians. The best results in that direction have been obtained by Fathi, Figalli and Rifford in [9], extending earlier results of Mather, see [15] (see also [18]). Their result imply that, if the dimension of $M$ is one or two, and if $L$ is sufficiently smooth ( $C^{4}$ is enough), then 3 hold. Extending this result in higher dimension, even for analytic Lagrangians, is a formidable problem. 


\section{A. The Conley structure of flows}

This section recalls some standard facts on the topological structure of flows on compact sets, due to Conley, see [7], see also [17], for example, for the extension to semi-flows. We provide the proof of some less standard statements which are useful in the present paper. It is convenient to work in an ambient metric space $(E, d)$. Let $X$ be a compact subset of $E$. A flow on $X$ is a continuous map $\varphi(t, x)=\varphi^{t}(x): \mathbb{R} \times X \longrightarrow X$ such that

$$
\varphi^{t} \circ \varphi^{s}=\varphi^{t+s}
$$

for all $s$ and $t$ in $\mathbb{R}$. A semi-flow on $X$ is a continuous map $\varphi(t, x)=\varphi^{t}(x)$ : $[0, \infty) \times X \longrightarrow X$ which satisfies the same relation for $s \geqslant 0$ and $t \geqslant 0$. We say that the subset $Y \subset X$ is positively invariant by the semi-flow $\varphi$ if $\varphi^{t}(Y) \subset Y$ for each $t \geqslant 0$. We say that $Y$ is invariant if it is positively invariant and if, in addition, for each $y \in Y$ and $t \geqslant 0$, there exists $z \in Y$ such that $\varphi^{t}(z)=y$. An $(\epsilon, T)$-chain of the semi-flow $\varphi^{t}$ is a piecewise continuous curve $x(t):[0, S] \longrightarrow X$ with finitely many times of discontinuity $S_{1}, \ldots, S_{k} \in[0, S]$ such that $x(t)=\varphi^{t-S_{i}}\left(x\left(S_{i}^{+}\right)\right)$for $\left.t \in\right] S_{i}, S_{i+1}[$, such that $S_{i+1} \geqslant S_{i}+T$ and such that $d\left(x\left(S_{i}^{-}\right), x\left(S_{i}^{+}\right)\right) \leqslant \epsilon$ for each $i$.

Definition 12. We say that $x \mathfrak{C}_{X} y$ (or, if there is no ambiguity, $x \mathfrak{C} y$ ) if, for each $\epsilon>0$ and $T>0$, there exists an $(\epsilon, T)$-chain $x(t):[0, S] \longrightarrow X$ such that $x(0)=x$ and $x(S)=y$.

The relation $\mathfrak{C}$ is closed. It is not hard to see that we have

$$
\varphi^{t}(x) \mathfrak{C} \varphi^{s}(y)
$$

for all $t \geqslant 0$ and $s \geqslant 0$ if $x \mathfrak{C} y$. This relation is satisfied for all $t$ and $s$ if $\varphi$ is a flow.

A points $x$ such that $x \mathfrak{C} x$ is called chain recurrent. On the set of chain recurrent points, the relation $\mathfrak{C}^{s}$ defined by

$$
x \mathfrak{C}^{s} y \Longleftrightarrow(x \mathfrak{C} y \quad \text { and } \quad y \mathfrak{C} x)
$$

is an equivalence relation. Its classes of equivalence are called the chain components of $X$. A semi-flow is said chain-recurrent if all its points are chain-recurrent, it is called chain-transitive if, in addition, it has only one chain component. The chain recurrent set of a semi-flow $\left(X, \varphi^{t}\right)$ is contained in $X_{\infty}:=\cap_{t \geqslant 0} \varphi^{t}(X)$. Moreover, by Lemma 18 below, the chain-recurrent set of the semi-flow $\left(X, \varphi^{t}\right)$ is the same as the chain recurrent set of the restricted semi-flow $\left(X_{\infty}, \varphi^{t}\right)$. The following is classical (see [17]): 
Proposition 13. Let $Y \subset X$ be the chain recurrent set of the semi-flow $\left(X, \varphi^{t}\right)$. Then $Y$ is a compact invariant subset of $X$ which is internally chain-recurrent, which means that the semi-flow $\left(Y, \varphi_{\mid Y}^{t}\right)$ is chain recurrent. The chain components of $\left(Y, \varphi_{\mid Y}^{t}\right)$ are the chain components of $\left(X, \varphi^{t}\right)$, they are the connected components of $Y$. Each chain component $Z \subset Y$ is internally chain-transitive, in the sense that $\left(Z, \varphi_{\mid Z}^{t}\right)$ is chain-transitive.

Definition 14. Let $x_{k}(t):\left[0, T_{k}\right] \longrightarrow E$ be a sequence of (not necessarily continuous) maps. We say that $x_{k}$ accumulates locally uniformly on the semi-flow $\left(X, \varphi^{t}\right)$ if for each $\epsilon>0$ and $T>0$, the following property holds for infinitely many $k \in \mathbb{N}$ :

$\forall S \in[0, \infty), \exists y \in X$ s. $t . d\left(x_{k}(t+S), \varphi^{t}(y)\right) \leqslant \epsilon \quad \forall t \in[0, T] \cap\left[0, T_{k}-S\right]$.

It is equivalent to say that, for each sequence $S_{k} \in\left[0, T_{k}\right]$, the sequence of curves $x_{k}\left(t+S_{k}\right)$ has a subsequence which converges uniformly on compact subsets of $[0, \infty)$ to a trajectory of $\varphi^{t}$.

We say that a sequence $\left(X_{k}, \varphi_{k}^{t}\right)$ of compact sets with semi-flows accumulates on $\left(X, \varphi^{t}\right)$ if for each $\epsilon>0$ and $T>0$, the following property holds for infinitely many $k \in \mathbb{N}$ :

$$
\forall x \in X_{k}, \exists y \in X \text { s. t. } d\left(\left(\varphi_{k}^{t}(x), \varphi^{t}(y)\right) \leqslant \epsilon \quad \forall t \in[0, T] .\right.
$$

It is equivalent to say that, for each $T>0$, each sequence $x_{k}(t):[0, T] \longrightarrow X_{k}$ of orbits of $\varphi_{k}^{t}$ has a subsequence which converges uniformly to an orbit of $\varphi^{t}$ in $X$.

Lemma 15. Let $x_{k}(t):\left[0, T_{k}\right] \longrightarrow E$ be a sequence of (not necessarily continuous) curves accumulating locally uniformly on the semi-flow $\left(X, \varphi^{t}\right)$. If in addition we have $x_{k}(0) \longrightarrow x$ and $x_{k}\left(T_{k}\right) \longrightarrow y$, then $x \mathfrak{C}_{X} y$.

Proof. Let us fix $\epsilon>0$ and $T>0$. We want to prove the existence of an $(\epsilon, T)$-chain between $x$ and $y$. Since the curves $x_{k}$ accumulates locally uniformly on the flow, there exists $k \in \mathbb{N}$ such that

$\forall i \in \mathbb{N}, \exists y_{i} \in X$ s. t. $d\left(x_{k}(t+i T), \varphi^{t}\left(y_{i}\right)\right) \leqslant \epsilon / 2 \quad \forall t \in[0, T] \cap\left[0, T_{k}-i T\right]$.

Let $y(t):\left[0, T_{k}\right] \longrightarrow X$ be the curve defined on each interval $[i T,(i+1) T[$ $\cap\left[0, T_{k}\right]$ by the expression $y(t)=\varphi^{t-i T}\left(y_{i}\right)$. We have

$$
\begin{aligned}
d\left(y\left(i T^{-}\right), y\left(i T^{+}\right)\right) & =d\left(\varphi^{T}\left(y_{i-1}\right), y_{i}\right) \\
& \left.\leqslant d\left(\varphi^{T}\left(y_{i-1}\right), x_{k}(i T)\right)+d\left(x_{k}(i T), y_{i}\right)\right) \leqslant \epsilon
\end{aligned}
$$

so that the curve $y(t)$ is an appropriate pseudo-orbit. 
Lemma 16. Let $\left(X_{k}, \varphi_{k}^{t}\right)$ be a sequence of compact semi-flows accumulating on $\left(X, \varphi^{t}\right)$. If $x_{k} \in X_{k}$ and $y_{k} \in X_{k}$ are sequences such that $x_{k} \longrightarrow x$, $y_{k} \longrightarrow y$ and $x_{k} \mathfrak{C}_{X_{k}} y_{k}$, then $x \mathfrak{C}_{X} y$.

Proof. Let $x_{k}(t):\left[0, T_{k}\right] \longrightarrow X_{k}$ be a sequence of curves such that each $x_{k}(t)$ is a $(1 / k, k)$-pseudo-orbit of $\varphi_{k}^{t}$ satisfying $x_{k}(0)=x_{k}$ and $x_{k}\left(T_{k}\right)=y_{k}$. We claim that the curves $x_{k}$ accumulate locally uniformly on $\left(X, \varphi^{t}\right)$. The conclusion then follows from Lemma 15. In order to prove the claim, let us fix $\epsilon>0$ and $T>0$. There exists $\delta \in] 0, \epsilon[$ such that, for all $x$ and $y$ in $X$ satisfying $d(x, y) \leqslant \delta$, and for all $t \in[0,4 T]$, we have $d\left(\varphi^{t}(x), \varphi^{t}(y)\right) \leqslant \epsilon / 4$. There are infinitely many values of $k>4 \max (T, 1 / \delta)$ such that

$$
\forall x \in X_{k}, \exists y \in X \text { s. t. } d\left(\left(\varphi_{k}^{t}(x), \varphi^{t}(y)\right) \leqslant \delta / 4 \quad \forall t \in[0,4 T] .\right.
$$

For these values of $k$, we claim that

$\forall S \in[0, \infty), \exists y \in X$ s. t. $d\left(x_{k}(t+S), \varphi^{t}(y)\right) \leqslant \epsilon \quad \forall t \in[0, T] \cap\left[0, T_{k}-S\right]$.

In order to prove the claim, notice that, given $S \geqslant 0$, the curve $x_{k}(t+S)$ has at most one jump on the interval $[0, T] \cap\left[0, T_{k}-S\right]$. As a consequence, there exists a time $\tau \in[0, T] \cap\left[0, T_{k}-S\right]$, such that $x_{k}(t+S)=\varphi_{k}^{t}(z)$ on $t \in[0, \tau] \cap\left[0, T_{k}-S\right]$, and $x_{k}(t+S)=\varphi_{k}^{t-\tau}(w)$ on $t \in[\tau, T] \cap\left[0, T_{k}-S\right]$ for some points $z$ and $w$ in $X_{k}$ which satisfy $d\left(\varphi_{k}^{\tau}(z), w\right) \leqslant 1 / k \leqslant \delta / 4$. There exist two points $Z$ and $W$ in $X$ such that

$$
d\left(\left(\varphi_{k}^{t}(z), \varphi^{t}(Z)\right) \leqslant \delta / 4 \quad \forall t \in[0,2 T]\right.
$$

and

$$
d\left(\left(\varphi_{k}^{t}(w), \varphi^{t}(W)\right) \leqslant \delta / 4 \quad \forall t \in[0,2 T] .\right.
$$

In particular, we have

$$
d\left(\varphi^{\tau}(Z), W\right) \leqslant d\left(\varphi^{\tau}(Z), \varphi_{k}^{\tau}(z)\right)+d\left(\varphi_{k}^{\tau}(z), w\right)+d(w, W) \leqslant \delta .
$$

Let us set $y:=\varphi^{-\tau}(Z)$. For each $t \in[0, \tau]$, we have

$$
d\left(x_{k}(t+S), \varphi^{t}(Z)\right)=d\left(\varphi_{k}^{t}(z), \varphi^{t}(Z)\right) \leqslant \delta / 4 \leqslant \epsilon,
$$

and for each $t \in[\tau, T] \cap\left[0, T_{k}-S\right]$, we have

$$
\begin{aligned}
d\left(x_{k}(t+S), \varphi^{t}(Z)\right) & =d\left(\varphi_{k}^{t-\tau}(w), \varphi^{t-\tau}\left(\varphi^{\tau}(Z)\right)\right) \\
& \leqslant d\left(\varphi^{t-\tau}(w), \varphi^{t-\tau}(W)\right)+d\left(\varphi^{t-\tau}(W), \varphi^{t-\tau}\left(\varphi^{\tau}(Z)\right)\right) \\
& \leqslant \delta / 4+\epsilon / 4 \leqslant \epsilon .
\end{aligned}
$$

This proves the claim.

The following obvious remark will be useful:

Lemma 17. Assume that the semi-flows $\left(X_{k}, \varphi_{k}^{t}\right)$ accumulate on $(X, \varphi)$, and that $X_{k}$ converge to $Y$ for the Hausdorff metric. Then $Y$ is a compact positively invariant subset of $X$, and $\left(X_{k}, \varphi_{k}^{t}\right)$ accumulate on $\left(Y, \varphi^{t}\right)$. 
If $\left(X, \varphi^{t}\right)$ is a semi-flow, then, for $k \in \mathbb{N}$, the set $X_{k}:=\varphi^{k}(X)$ is positively invariant, so that $\left(X_{k}, \varphi^{t}\right)$ is itself a semi-flow. It is not hard to see that the relations $\mathfrak{C}_{X}$ and $\mathfrak{C}_{X_{k}}$ coincide on $X_{k}$. Defining now $X_{\infty}:=\cap_{k \in \mathbb{N}} X_{k}$ we have:

Lemma 18. The set $X_{\infty}$ is positively invariant; the relations $\mathfrak{C}_{X}$ and $\mathfrak{C}_{X_{\infty}}$ coincide on $X_{\infty}$.

Proof. The relations $\mathfrak{C}_{X_{k}}, k \in \mathbb{N}$ all coincide on $X_{\infty}$. We have to prove that

$$
x \mathfrak{C}_{X_{k}} y \Rightarrow x \mathfrak{C}_{X_{\infty}} y .
$$

This follows from Lemma 16 since the semi-flows $\left(X_{k}, \varphi^{t}\right)$ accumulate on $\left(X_{\infty}, \varphi^{t}\right)$.

Acknowledgements. I thank Albert Fathi for pointing out that $5 \Rightarrow 4$ in Section 3.

\section{References}

[1] Bernard, P.: Homoclinic orbits to invariant sets of quasi-integrable exact maps. Ergodic Theory Dynam. Systems 20 (2000), no. 6, 1583-1601.

[2] Bernard, P.: Connecting orbits of time dependent Lagrangian systems. Ann. Inst. Fourier (Grenoble) 52 (2002), 1533-1568.

[3] Bernard, P.: Existence of $C^{1,1}$ critical sub-solutions of the HamiltonJacobi equation on compact manifolds. Ann. Sci. École Norm. Sup. (4) 40 (2007), no. 3, 445-452.

[4] Bernard, P.: The dynamics of pseudographs in convex Hamiltonian systems. J. Amer. Math. Soc. 21 (2008), 615-669.

[5] Bernard, P. And Buffoni, B.: Weak KAM pairs and MongeKantorovich duality. In Asymptotic analysis and singularities - elliptic and parabolic PDEs and related problems, 397-420. Adv. Stud. Pure Math. 47-2. Math. Soc. Japan, Tokyo, 2007.

[6] Bernard, P. and Contreras, G.: Generic properties of families of Lagrangian systems. Ann. of Math. (2) 167 (2008), no. 3, 1099-1108.

[7] Conley, C.: The gradient structure of a flow. I. With a comment by R. Moeckel. Ergodic Theory Dynam. Systems 8 (1988). Charles Conley Memorial Issue, 11-26.

[8] Contreras, G.: Action potential and weak KAM solutions. Calc. Var. Partial Differential Equations 13 (2001), no. 4, 427-458.

[9] Fathi, A., Figalli, A. And Rifford, L.: On the Hausdorff dimension of the Mather quotient. Comm. Pure Appl. Math. 62 (2009), no. 4, 445-500.

[10] FATHI, A.: Weak KAM Theorem in Lagrangian Dynamics. Book to appear. 
[11] MAÑé, R.: Generic properties and problems of minimizing measures of Lagrangian systems. Nonlinearity 9 (1996), no. 2, 273-310.

[12] MAÑÉ, R.: Lagrangian flows: the dynamics of globally minimizing orbits. Bol. Soc. Brasil. Mat. (N.S.) 28 (1997), 141-153.

[13] Mather, J. N.: Action minimizing invariant measures for positive definite Lagrangian systems. Math. Z. 207 (1991), 169-207.

[14] Mather, J. N.: Variational construction of connecting orbits. Ann. Inst. Fourier (Grenoble) 43 (1993), 1349-1368.

[15] Mather, J. N.: Total disconnectedness of the quotient Aubry set in low dimensions. Comm. Pure Appl. Math. 56 (2003), 1178-1183.

[16] Mather, J. N.: Examples of Aubry sets Ergod. Theory Dynam. Systems 24 (2004), 1667-1723.

[17] Patrão, M. and San Martin, L. A. B.: Semiflows on topological spaces: chain transitivity and semigroups. J. Dynam. Differential Equations 19 (2007), no. 1, 155-180.

[18] Sorrentino, A.: On the total disconnectedness of the quotient Aubry set. Ergodic Theory Dynam. Systems 28 (2008), no. 1, 267-290.

Recibido: 5 de marzo de 2008

Revisado: 11 de septiembre de 2008

Patrick Bernard

Université Paris-Dauphine

CEREMADE, UMR CNRS 7534

Pl. du Maréchal de Lattre de Tassigny

75775 Paris Cedex 16, France patrick. bernard@ceremade.dauphine.fr 